\title{
Worse or even better than expected? Outcome expectancies and behavioral experiences in the context of physical activity among cancer patients
}

Journal of Health Psychology 202I, Vol. 26(5) 659-67I (C) The Author(s) 2019 Article reuse guidelines: sagepub.com/journals-permissions DOI: $10.1177 / 1359105319832345$ journals.sagepub.com/home/hpq @SAGE

\author{
Nadine Ungar' (iD, Fiona S Rupprecht', \\ Karen Steindorf ${ }^{2,3}$, Joachim Wiskemann ${ }^{3,4}$ \\ and Monika Sieverding'
}

\begin{abstract}
Expectancies of cancer patients regarding their physical activity before they took part in a behavior change intervention were compared with their experiences during the intervention period. A total of 66 cancer patients completed either a randomly assigned 4-week physical activity or a stress-management counseling intervention. On average, participants had positive expectancies toward physical activity. Outcome expectancies predicted outcomes (e.g. physical activity) at a 10-week follow-up. Outcome realization (discrepancy between expectancies and experiences) further increased explained variance in self-efficacy and physical activity enjoyment. In conclusion, not only initial outcome expectancies but also their realizations seem to be important for subsequent behavior and cognitions.
\end{abstract}

\section{Keywords}

behavior change, behavioral experiences, cancer, outcome expectancies, physical activity

An increasing number of cancer survivors are facing the burden of cancerous diseases (International Agency for Research on Cancer, 2017). Over the past 20 years, strong evidence has been accumulated for beneficial effects of regular physical activity during cancer treatment on diverse health outcomes such as reducing treatment-related side effects, physical functioning, and increasing quality of life (see for reviews Buffart et al., 2017; Mishra et al., 2012). There is even first evidence that physical activity might lead to a prolonged life expectation among cancer patients (Cormie et al., 2017). Despite this high potential, only approximately one-third of cancer survivors are meeting physical activity guidelines (Crawford et al., 2016).

To understand this gap, we wanted to find out what insufficiently active cancer patients expect regarding physical activity. Expectations play a key role in forming an individual's behavior and

\footnotetext{
'Heidelberg University, Germany

${ }^{2}$ German Cancer Research Center (DKFZ), Germany

${ }^{3}$ National Center for Tumor Diseases (NCT), Germany

${ }^{4}$ Heidelberg University Hospital, Germany

Corresponding author:

Nadine Ungar, Institute of Psychology, Heidelberg

University, Hauptstraße 47-5I, 691 17 Heidelberg, Germany.

Email: nadine.ungar@alumni.uni-heidelberg.de
} 
cognitions, and are a core concept in social cognitive models such as Bandura's Social Cognitive Theory (SCT, Bandura, 1986) and the Health Action Process Approach (HAPA, Schwarzer, 2008). According to these theories, individuals have beliefs about the consequences of a behavior, that is, that it leads to (un)desired outcomes, which can in turn influence their (intention and) behavior. These beliefs are called outcome expectancies. For example, the belief that physical activity leads to a better well-being can motivate people to engage in physical activity. There is empirical evidence that outcome expectancies can explain the adoption and maintenance of health behaviors (e.g. Nudelman and Shiloh, 2018). However, in a meta-analysis by Young et al. (2014) on the effects of SCT variables only a third of the 71 included outcome expectancy constructs showed direct effects on physical activity.

In the context of a severe disease such as cancer, outcome expectancies might be especially relevant. A cancer diagnosis is often described as teachable moment (DemarkWahnefried et al., 2005) and can be connected with high expectations (Hoffmann and Del Mar, 2015). Previous research has applied a range of different social cognitive models and found that cancer survivors had both unique exercise motives that are related to their cancer experience and motives known from the general population (see Vallance and Courneya, 2012 for an overview). Studies applying the SCT found associations between outcome expectancies and exercise for different cancer populations (e.g. Coups et al., 2009; Endrighi et al., 2016).

In contrast to outcome expectancies, behavioral experiences with a health behavior (Rothman, 2000) and outcome realizationthat is, the accordance between outcome expectancies and behavioral experiences (Wilcox et al., 2006) - have not gotten much attention in previous research (exceptions are Loehr et al., 2014; Neff and King, 1995; Sears and Stanton, 2001; Wilcox et al., 2006). Specifically, physical activity as well as other health behaviors might be better (e.g. more joyful, safer, and less exhausting) or worse (e.g. more boring and more difficult to perform) than expected. A study by Wilcox et al. (2006) with 118 women aged around 65 years revealed that outcome realization and not initial outcome expectancies predicted participants' physical activity at 6-month follow-up. In addition, the study by Wilcox et al. (2006) went one step further as it examined a combination of baseline outcome expectancies and outcome realization (similar to Neff and King, 1995). Four expectation groups were created (pessimistic realists, optimistic realists, surprised pessimists, and disappointed optimists) by allocating participants according to their initial outcome expectancies (low vs high) and their achievement at 6 months (low vs high). By longitudinally examining older women's physical activity, results from 7 to 12 months follow-up revealed that surprised pessimists (low expectations and high achievement) and optimistic realists (high expectations and high achievement) had the highest physical activity levels.

Similar to outcome expectancies, the discrepancy between outcome expectancies and behavioral experiences (=outcome realization) might be more pronounced in the oncological context, as people are in a new (disease) situation. The development of the disease and possible side effects are often unpredictable for the patients (e.g. Weeks et al., 2012), thus expectancies might be less accurate. However, to our knowledge, outcome realization has not been studied among cancer patients so far. Our study examined the role of outcome expectancies and outcome realization in the context of a behavior change intervention with cancer patients (MOTIVACTION study, Ungar et al., 2016a) based on the HAPA (Schwarzer, 2008). We extended the research by Wilcox et al. (2006) by incorporating it in the HAPA framework. Thereby, we examined not only trajectories in physical activity, but also in other core HAPA variables such as self-efficacy and intention as potential correlates and long-term determinants of physical activity (Schwarzer, 2008). According to the HAPA, outcome expectancies are (among other variables) supposed to predict intention, which in turn 
is - together with maintenance self-efficacy-a predictor of the actual behavior. In addition, the HAPA framework includes resources such as social support as potential moderators. Physical activity enjoyment was added as an affective component based on previous research highlighting the important role of affective factors in addition to the before mentioned cognitive variables (Klusmann et al., 2016; Ungar et al., 2016b).

In summary, as only a small fraction of cancer patients meet physical activity guidelines (Crawford et al., 2016) despite its beneficial health outcomes (Buffart et al., 2017), healthcare professionals should be informed how to adequately counsel their patients toward behavior change. Thereby, addressing cancer patients' expectancies and experiences might be a key element to initiate and adopt an active lifestyle. Being - to our knowledge - the first study investigating outcome realization in cancer patients, this study examined the effects of outcome expectancies and outcome realization regarding physical activity in a behavior change intervention with cancer patients. We expected that initial outcome expectancies and outcome realization would predict participants' intention, self-efficacy, physical activity enjoyment, and meeting of physical activity guidelines at follow-up. Furthermore, we examined if pessimistic realists, optimistic realists, surprised pessimists, and disappointed optimists differed in their changes between baseline and follow-up in these variables.

\section{Methods}

\section{Participants}

Patients of any cancer entity were recruited at the National Center for Tumor Diseases in Heidelberg, Germany. Inclusion criteria were the following: being $\geqslant 18$ years, receiving currently outpatient treatment or having finished this treatment no longer than 6 months ago, not meeting the exercise guidelines of $150 \mathrm{~min} /$ week, and being able to follow the study instructions. Exclusion criteria were the following: wound healing not completed, bone metastases, rehabilitation or inpatient treatment planned for the next 8 weeks, and serious comorbidities or comorbidity-related limitations.

Out of 72 cancer patients who initially took part in the study, 67 completed it, yielding a dropout rate of 6.9 percent (a flow chart of the MOTIVACTION study as well as detailed sample characteristics can be seen in Ungar et al., 2016a). One further participant was excluded from the analysis as this person did not report any (experiences with) physical activity during the study period. Thus, the final sample consisted of 66 cancer patients $\left(M_{\mathrm{age}}=54.8\right.$ years; $53.0 \%$ female) with the majority having (had) a breast cancer diagnosis $(31.8 \%)$ followed by colorectal cancer $(13.6 \%)$ and prostate cancer (7.6\%). More than a third $(37.9 \%)$ currently received chemotherapy and 13.6 percent were currently treated with radiotherapy. Metastases were reported by 34.8 percent. These illnessrelated characteristics along with sociodemographic variables were assessed at $\mathrm{T} 1$ via self-report and are depicted in the Supplementary Table S1.

\section{Design and procedure}

The MOTIVACTION study has already been described elsewhere (Ungar et al., 2016a). In short, patients were randomized (stratified by sex, age, metastases, and chemotherapy status) to a 4-week exercise or a 4-week stress management intervention. Both interventions started with an individual 1-hour-counseling session that focused on increasing self-regulation toward exercise/stress management based on the HAPA (e.g. goal setting, action planning, and goal pursuing). Participants should practice at home during a 4-week period being supported by diverse materials (e.g. booklet and CD). During these 4 weeks, participants received three telephone calls (lasting about 15 minutes) in which the past week was reviewed, barriers were addressed, and goals and plans were adapted if necessary. Participants of both intervention groups were kept in the analyses if they reported any behavioral experiences with 
physical activity during the intervention phase, as previous analyses found significant increases in physical activity not only in the exercise group but also among participants practicing stress management (Ungar et al., 2016a). Assessment points were prior to the intervention (T1), directly after the 4-week long intervention (T2), and 10 weeks after the intervention was finished (T3). All subjects gave written informed consent (ethic committee of the medical faculty from Heidelberg University, Germany; clinicalTrials.gov: NCT01576107).

\section{Measures}

Outcome expectancies and behavioral experiences. Expectancies on the outcomes of physical activity were only assessed prior to the intervention (T1) when participants were inactive or insufficiently active. Behavioral experiences were measured after the 4-week intervention (T2) in order to capture how the experiences with physical activity relate to the initial expectancies. Both constructs were assessed with 14 items each, based on scales by Fuchs (Fuchs, 1994, 2013). To adapt these scales to the oncological context, a qualitative interview was conducted with 60 cancer patients to assess what they expect regarding physical activity in an open format. The most frequently mentioned positive $(N=13)$ and negative $(N=13)$ expectancies were included in a quantitative pretest with 63 cancer patients, in which item statistics and internal consistencies were calculated. Based on these results, unique expectancies capturing the cancer experiences $(N=6)$ gained through the pretest were combined with eight original items of the scales by Fuchs. All items are listed in Table 1. Responses ranged from 1 (not true) to 4 (completely true) and the negatively framed items were reversely coded for calculating means and outcome realization. The internal consistencies were adequate for both scales (Cronbach's $\alpha_{\text {expectancies }}=.72$, Cronbach's $\alpha_{\text {experiences }}=.74$ ).

Outcome realization. Outcome realization was calculated by subtracting outcome expectancies from the actual behavioral experiences (similar to Sears and Stanton, 2001) as both variables were measured across the same categories with a focus on either expectancies or experiences. Thereby, positive values on outcome realization indicated that the experiences were better than the expectancies, and negative values indicated that the experiences were worse than the expectancies.

Behavioral intention. Behavioral intention was measured at all three measurement points with one item according to the HAPA (Schwarzer, 2008; Schwarzer et al., 2003). This item was "Please think of the next four weeks [T1]/next two months [T2]/next six months [T3]. Do you intend to be regularly physically active for at least 150 minutes (e.g. half an hour at 5 days) per week?" and the possible answers ranged from 1 (not true) to 4 (completely true).

Maintenance self-efficacy. Maintenance self-efficacy was assessed at all three measurement points according to the HAPA (Schwarzer, 2008; Schwarzer et al., 2003) and adapted to the oncological context based on a pretest. Seven items were used measuring the confidence in sticking to regular physical activity. An example item was "I am confident that I can be regularly physically active in the long term even if I experience side-effects (e.g. nausea) of the cancer therapy." Responses ranged from 1 (not true) to 4 (completely true). Cronbach's alpha was good $(\alpha=.88$ at $\mathrm{T} 1$ and $\mathrm{T} 3$ ).

Physical activity enjoyment. Physical activity enjoyment was measured at all three measurement points with two items based on Rogers et al. $(2011,2015)$. The items were "It is fun to engage in sport activities and regular physical activity" and "I enjoy being regularly physically active" (from $1=$ not true to $4=$ completely true). The internal consistency was good (Cronbach's $\alpha=.88$ at T1 and .83 at T3).

Physical activity. Physical activity was measured using a slightly modified Short Questionnaire to ASsess Health-enhancing Physical Activity (SQUASH, Wagenmakers et al., 2008; WendelVos et al., 2003) at all three time points. The questionnaire contained questions about physical 


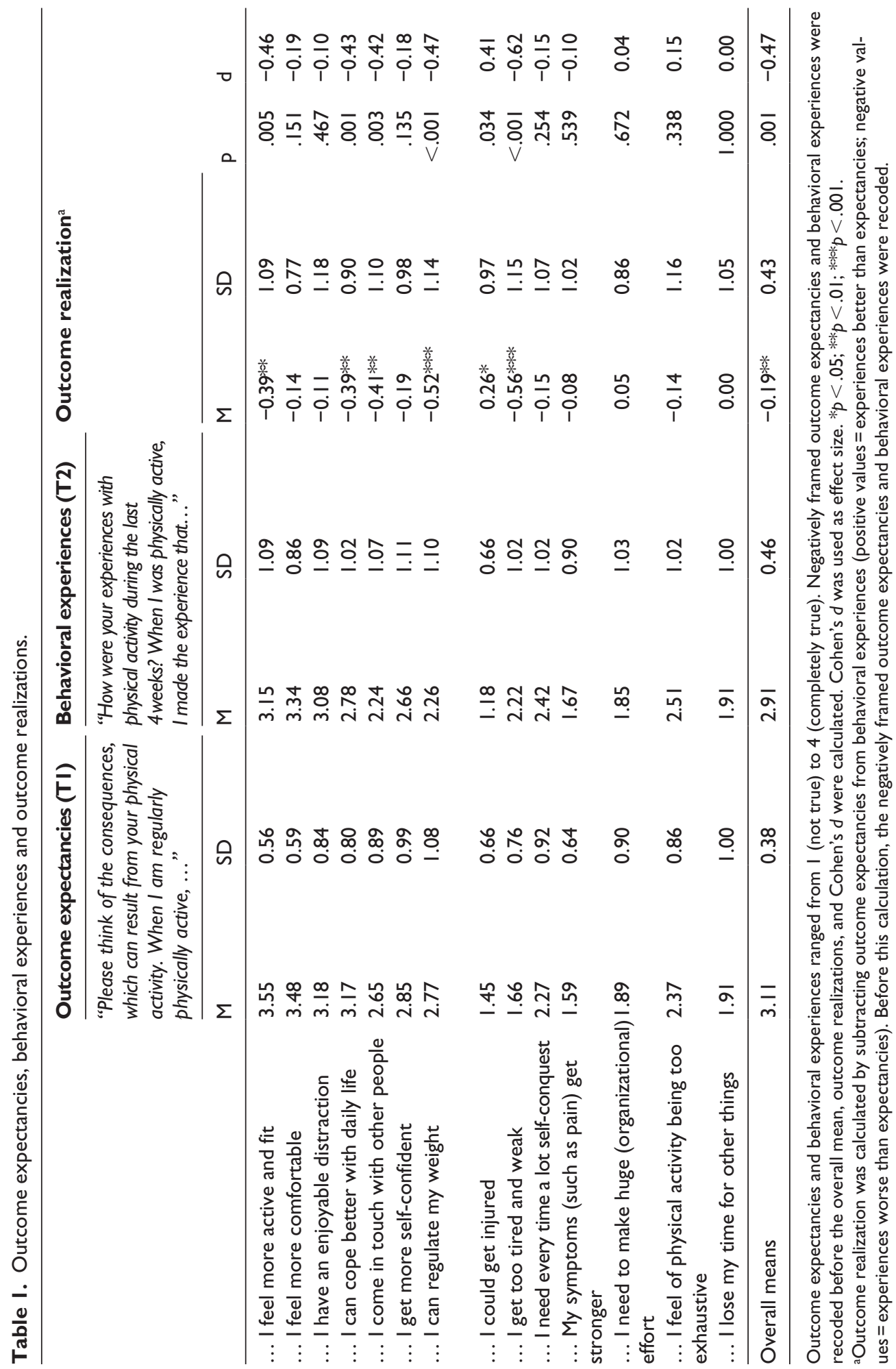


activities in four domains (commuting, household, leisure-time, and work) during the past 7 days, which were rated in terms of days per week, average time per day, and intensity (light, moderate, or vigorous). For our main analysis, we focused on at least moderate leisure-time activities as we were interested in intended physical activity rather than physical activity in general (e.g. household activities). The respective six items were the following: brisk (Nordic) walking, bicycling, gymnastics or resistance training, and three open-ended items called "sport activities," in which participants could fill in their own activities. In accordance with Ungar et al. (2016a), the variable of primary interest was "meeting physical activity guidelines" $(0=$ no; $1=y e s)$. The cutoff for the dichotomization was based on the official guidelines of $150 \mathrm{~min} /$ week (Schmitz et al., 2010). In the MOTIVACTION study, physical activity was additionally measured by accelerometer at T1 and T2. As this article focuses on physical activity maintenance assessed at the 10-week follow-up (T3), the accelerometer data could not be included in the main analyses. However, as reported in Ungar et al. (2016a), selfreported physical activity was validated with accelerometer measures. For example, for moderate-to-vigorous physical activity, self-reported and accelerometer-based measures revealed a correlation of $r=.71(p<.01)$.

Social support for exercise. Social support for exercise was assessed with six items based partly on Sallis et al. (1987) at all three measurement points. Participants were asked how often a family member (item 1)/a friend (item 2)/another person (item 3 ) has exercised with them and how often a family member (item 4)/a friend (item 5)/another person (item 6) offered to exercise with them. Responses ranged from 1 (never) to 5 (very often). Cronbach's alpha was decent for T1 $(\alpha=.61)$, but good for T3 $(\alpha=.85)$.

\section{Statistical analyses}

In a first step, we calculated paired $t$-tests in order to assess item-specific discrepancies between outcome expectancies and behavioral experiences. For the main analyses, we calculated hierarchical regression analyses in order to test whether outcome experiences and/or outcome realization predicted intention, self-efficacy, and physical activity enjoyment (assessed at T3). For the same purpose, a logistic hierarchical regression was used to predict if physical activity guidelines were met at T3. In the first block of the hierarchical regressions, the baseline score of the respective dependent variable (T1) was entered together with all those control variables (sociodemographic and illness-related variables, as well as intervention group, that is, exercise or stress management) that were significantly correlated with the dependent variable on a bivariate level. Outcome expectancies were entered, additionally, in step 2 and outcome realization was entered, additionally, in step 3. Behavioral experiences were not included in the regression, as the simultaneous consideration of outcome expectancies, behavioral experiences, and outcome realization would have led to singularity.

To further illustrate the importance of combining initial outcome expectancies and behavioral experiences in analyses, we assigned the study participants to four outcome realization groups. Following a procedure by Neff and King (1995) and Wilcox et al. (2006), patients were divided into these four groups using median splits on outcome expectancies and behavioral experiences: pessimistic realists were defined as participants who had outcome expectancies and behavioral experiences below the sample median, optimistic realists had comparatively high outcome expectancies and high behavioral experiences, disappointed optimists scored above the sample median for outcome expectancies but below the median for behavioral experiences, and surprised pessimists had comparatively low outcome expectancies but higher behavioral experiences during the intervention. Baseline and follow-up scores in intention, self-efficacy, and physical activity enjoyment of the four outcome realization groups were compared using paired $t$-tests. The four groups were compared regarding "meeting physical activity guidelines" using a chi-square test.

Finally, an explorative analysis was performed to find a preliminary explanation for the 
differences between the four groups regarding the outcome variables and especially, the success of the surprised pessimists. Therefore, baseline and T2 social support were compared within the four groups using paired $t$-tests. This allowed us to identify if single outcome realization groups experienced declines or increases in social support during the intervention period.

\section{Results}

\section{Outcome expectancies and behavioral experiences}

Mean values of outcome expectancies and behavioral experiences are depicted in Table 1. Overall, participants had positive initial expectancies toward physical activity $(M=3.11$, from 1 (negative) to 4 (positive)). Behavioral experiences tended to be less positive than the corresponding expectancies $(M=2.91)$. Hence, there was a trend to have a negative outcome realization, $t(65)=3.63, p=.001, d=-0.47$. For six items, outcome expectancies significantly differed from behavioral experiences with small to moderate effect sizes. Specifically, cancer patients reported - on average - to get more tired and weak (due to physical activity) than expected $(d=-0.62)$, that they could regulate their weight (by physical activity) less than expected $(d=-0.47)$, and that they felt less active and fit than expected $(d=-0.46)$. In addition, physical activity helped them less than expected to handle their daily lives $(d=-0.43)$, and they came in touch with others (through physical activity) less than expected $(d=-0.42)$. However, the cancer patients also reported to be injured less often than expected $(d=0.41)$.

\section{Outcome expectancies and outcome realization as predictors of intention, self-efficacy, physical activity enjoyment, and meeting physical activity guidelines}

Table 2 shows the results of the (logistic/linear) hierarchical regression analyses with outcome expectancies and outcome realization as predictors of intention, self-efficacy, physical activity enjoyment, and meeting physical activity guidelines at T3. Most potential control variables (age, sex, family status, education level, occupation, cancer entity, existence of metastases and treatment status regarding surgery and radiotherapy, as well as intervention group) were unrelated to the T3 outcome variables. Only chemotherapy status correlated significantly with intention, self-efficacy, and meeting physical activity guidelines and was thus entered in the first step of the regression together with the baseline value of the respective outcome variable (e.g. intention T1 as control variable for predicting intention T3). Outcome expectancies were entered in addition to the control variables in the second step of the regression and were a marginally significant predictor of intention $(\beta=.24, p=.058)$, and a significant predictor of self-efficacy $(\beta=.24$, $p=.048)$, physical activity enjoyment $(\beta=.41$, $p=.008)$, and meeting physical activity guidelines (yes/no) $(B=1.85, p=.033)$. Thus, individuals with more positive outcome experiences showed a higher intention, self-efficacy, and physical activity enjoyment and were more likely to meet physical activity guidelines at follow-up even after controlling for baseline scores. The results of our study thus strengthened prior findings on the important role of outcome expectancies for a vast array of outcomes in the context of physical activity (Young et al., 2014). In the third step of the regression, outcome realization was added as the last predictor and explained 12.4 percent of additional variance in self-efficacy $(\beta=.39, p=.001)$ and 14.5 percent in physical activity enjoyment $(\beta=.43, p=.001)$. However, outcome realization did not have a significant effect on meeting physical activity guidelines and intention at follow-up. Hence, for some but not for all variables it was of importance if the outcome expectancies were met, exceeded, or missed.

\section{Group comparisons}

Differences between baseline and follow-up scores for pessimistic realists $(N=23)$, optimistic 
Table 2. Hierarchical regression analyses for the prediction of meeting physical activity guidelines, intention, self-efficacy, and physical activity enjoyment measured at I0-week follow-up (T3).

\begin{tabular}{|c|c|c|c|c|c|c|c|c|}
\hline & \multirow{2}{*}{\multicolumn{2}{|c|}{$\begin{array}{l}\text { Intention } \\
\mathrm{N}=64\end{array}$}} & \multirow{2}{*}{\multicolumn{2}{|c|}{$\begin{array}{l}\text { Self-efficacy } \\
N=64\end{array}$}} & \multirow{2}{*}{\multicolumn{2}{|c|}{$\begin{array}{l}\text { PA enjoyment } \\
N=64\end{array}$}} & \multirow{2}{*}{\multicolumn{2}{|c|}{$\begin{array}{l}\text { Meeting PA guidelines }^{\mathrm{a}} \\
\mathrm{N}=65\end{array}$}} \\
\hline & & & & & & & & \\
\hline & $\beta$ & $\Delta \mathrm{R}^{2}$ & $\beta$ & $\Delta \mathrm{R}^{2}$ & $\beta$ & $\Delta \mathrm{R}^{2}$ & $\beta$ & $\Delta \mathrm{R}^{2}$ \\
\hline Step I & & $.303 * * *$ & & $.314 * * *$ & & $.155^{* *}$ & & $.156^{*}$ \\
\hline Chemotherapy & $-.24 *$ & & $-.24 *$ & & & & $-1.17^{\circ}$ & \\
\hline Baseline level $\mathrm{TI}^{\mathrm{b}}$ & $.38 * *$ & & $.40 * *$ & & .15 & & .93 & \\
\hline Step 2 & & .032 & & .003 & & .020 & & $.066^{\circ}$ \\
\hline $\begin{array}{l}\text { Outcome } \\
\text { expectancies TI }\end{array}$ & $.24^{\circ}$ & & $.24^{*}$ & & $.4 I^{* *}$ & & $1.85^{*}$ & \\
\hline Step 3 & & .012 & & $.124 * *$ & & $.145^{* *}$ & & .023 \\
\hline Outcome realization & .12 & & $.39 * *$ & & $.43^{* *}$ & & .82 & \\
\hline Total $R^{2}$ & .346 & & .441 & & .319 & & .247 & \\
\hline Adjusted $R^{2}$ & .301 & & .403 & & .283 & & & \\
\hline$F\left(\chi^{2}\right.$ for physical activity) & $7.68 * * *$ & & $11.65 * * *$ & & $8.75^{* * *}$ & & $13.07^{*}$ & \\
\hline
\end{tabular}

PA: physical activity.

For intention, self-efficacy, and PA enjoyment, linear regression analyses were computed. For "meeting PA guidelines" a logistic regression analysis was computed. Predictors were entered to the models in three subsequent steps (i.e. in each step, the newly added, and all prior predictors are included). In addition, explained variances are given for steps 2 and 3.

a Meeting PA guidelines was coded as $0=$ not meeting PA guidelines of $150 \mathrm{~min} /$ week and $\mathrm{I}=$ meeting the guidelines of $150 \mathrm{~min} /$ week.

bThe respective baseline level of the outcome variable was used as a predictor (e.g. intention at baseline as predictor for intention at 10 -week follow-up).

${ }^{\circ} p<.08 ; * p<.05 ; * * p<.01 ; * * * p<.001$.

realists $(N=20)$, surprised pessimists $(N=13)$, and disappointed optimists $(N=10)$ are depicted in Figure 1. Baseline and follow-up scores differed most frequently among surprised pessimists. These participants with comparably low initial expectancies but positive behavioral experiences yielded significantly higher follow-up scores for intention $\left(M_{\mathrm{T} 1}=3.15\right.$ vs $\left.M_{\mathrm{T} 3}=3.64, t=2.31, p=.018, d=0.57\right)$, selfefficacy $\left(M_{\mathrm{T} 1}=2.55\right.$ vs $M_{\mathrm{T} 3}=2.92, t=2.67$, $p=.021, d=0.61)$, and physical activity enjoyment $\left(M_{\mathrm{T} 1}=2.71\right.$ vs $M_{\mathrm{T} 3}=3.21, t=2.57$, $p=.026, d=0.86)$. Regarding physical activity behavior, optimistic realists yielded the strongest effect: 60.0 percent reached the physical activity guidelines at follow-up compared with only 25.0 percent at baseline $\left(\mathrm{chi}^{2}=5.01\right.$, $p=.025, \breve{\omega}=0.50$ ).

\section{Additional analyses for surprised pessimists}

As surprised pessimists showed quite convincing success in several variables in the previous analyses, we further examined exploratively if social support could explain the "better than expected" outcome for this group. Increases in social support (e.g. by family, friends, or a role model) over the study period might be one reason why the initially negative experiences of surprised pessimists were exceeded during the intervention. Therefore, we examined if baseline social support increased during the received intervention for the four groups. Paired $t$-tests revealed that social support was perceived to considerably increase during the intervention (baseline compared with T2) only 


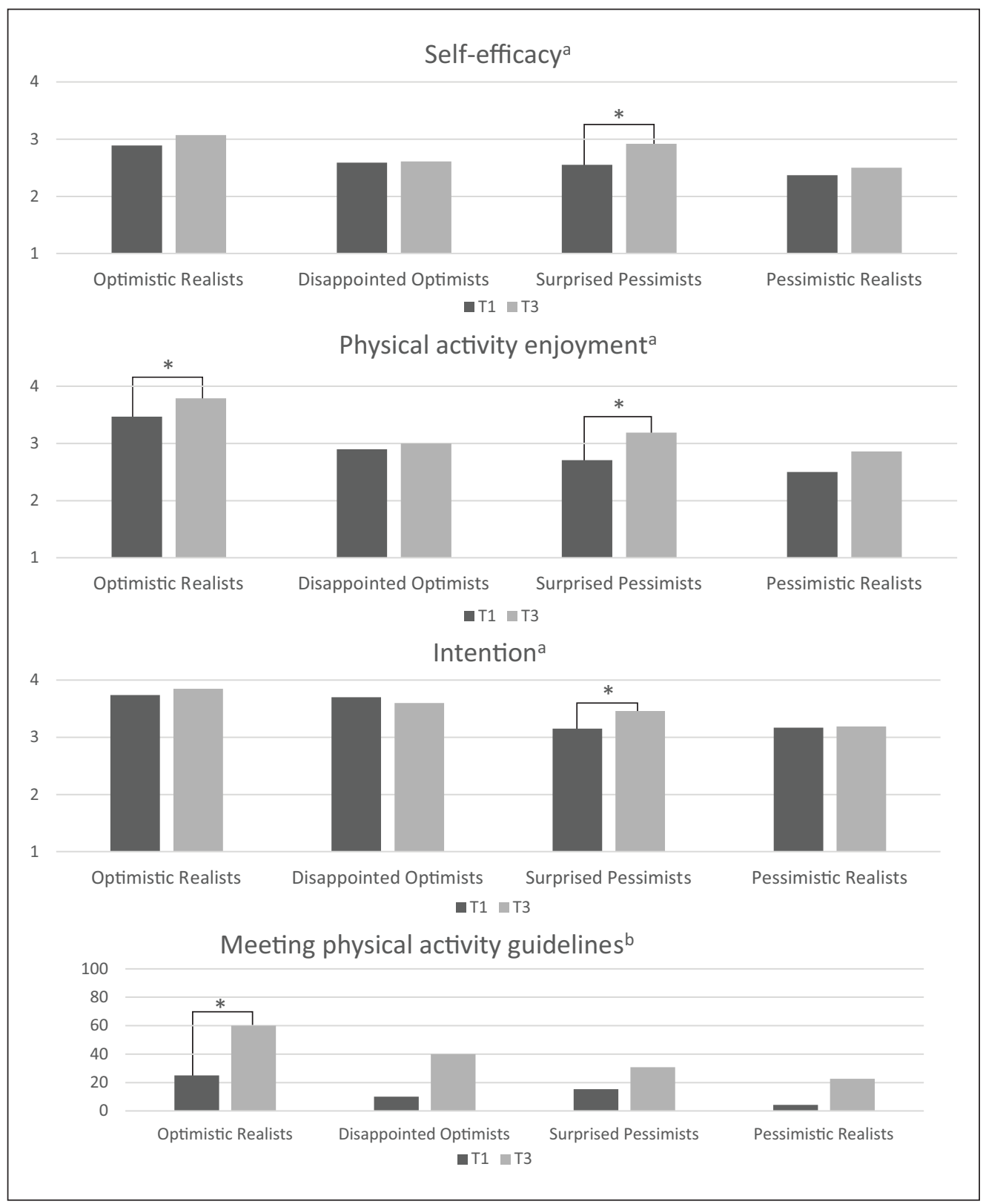

Figure I. Comparing optimistic realists, disappointed optimists, surprised pessimists, and pessimistic realists in their baseline and follow-up scores on different outcome variables.

aVariable ranges from I (not true) to 4 (completely true) and a paired $t$-test was calculated.

bPercentage of participants meeting the physical activity guidelines, chi-square tests were calculated.

$* p<.05$.

by surprised pessimists $\left(M_{\mathrm{T} 1}=1.97\right.$ vs three other groups did not report any signifi$\left.M_{\mathrm{T} 2}=2.55, t(12)=2.81, p=.016, d=0.87\right)$. The cant change in social support. 


\section{Discussion}

This study examined cancer patients' outcome expectancies toward physical activity and their realization during an intervention. Overall, cancer patients reported relatively positive expectancies. For example, they expected to feel more fit and comfortable, to have an enjoyable distraction and to cope better with daily life due to physical activity. As research on the various health benefits of physical activity during cancer treatment has just been established during the last few decades (see Courneya (2009) for a historical review), the positive expectancies of cancer patients are encouraging as research seems to have reached practice at least among our participants. As our participants were however interested in participating in a study on the topic of physical activity or stress management, their outcome expectancies may have been positively biased. Experiences patients made during the intervention period were - on average-(slightly) more negative. This might be the case as a cancer treatment is often connected with unexpected adverse-effects, and the disease itself develops in a way that might have been unforeseeable and might interfere with physical activity. In addition, physical activity is a strategy through which patients can actively deal with their disease themselves. The hope connected with this (which might also be pushed by the media, etc.) might be one reason for unrealistically high expectations at the beginning. However, some other patients' expectancies were relatively accurate, as they were mostly realized during the intervention period. This might have been supported by the psychological counseling as a major part of both interventions aiming at strengthening participants' self-regulation capacities.

Previous research on physical activity (and other health behaviors) rarely examined the accordance between outcome expectancies and behavioral experiences (= outcome realization) (Loehr et al., 2014; Neff and King, 1995; Sears and Stanton, 2001; Wilcox et al., 2006). However, the interplay between expectancies and experiences leads to a more fluid and dynamic conceptualization of social cognitive constructs (Loehr et al., 2014; Wilcox et al., 2006), as originally theorized by Bandura (1997). The more dynamic concept of outcome realization might help to explain the inconsistent findings regarding the association between initial outcome expectancies and physical activity found in previous studies (see metaanalysis by Young et al., 2014). In our study, outcome realization did not significantly predict if participants met physical activity guidelines at follow-up; outcome expectancies themselves, on the other hand, predicted physical activity. In theory, outcome expectancies are thought to influence especially the initiation of behavior (Schwarzer, 2008), whereas outcome realization is meant to be especially relevant for behavior maintenance (Rothman, 2000). In our study, outcomes were measured at a 10-week follow-up and it is not clear if this period is long enough to capture maintenance. Self-efficacy and physical activity enjoyment as potential predictors of physical activity (Ungar et al., 2016b) were, however, influenced by outcome realization in our study. This may suggest that an effect on physical activity might emerge in the more distant future.

Group profiles illustrated that the combination between the level of initial outcome expectancies and behavioral experiences seems to play a role. Our study replicated the finding by Wilcox et al. (2006) among older women. In addition, we extended this finding by including not only physical activity itself but also cognitive and affective factors. We found that surprised pessimists reported improvements not only in intention, physical activity enjoyment, and self-efficacy, but also in social support. Social support from family members, friends, or role models (as being a main component of the MOTIVACTION study) might have helped participants with low initial outcome expectancies to make nevertheless positive experiences during the intervention. Increasing the social support might thus be a starting point for future interventions addressing low outcome expectancies. 
Clinical implications and practical recommendations can be concluded from our results. It seems worthwhile that healthcare professionals assess patients' outcome expectancies before the start of an exercise program. Thereby, special attention has to be paid to unrealistically high expectations, as participants seem to slightly overestimate the benefits. Finally, our results have shown that outcome expectancies do not always match with the experience participants make, supporting the view that outcome expectancies are dynamically changing. Thus, it seems advisable to not only assess initial outcome expectancies but to additionally ask patients about their experiences and follow-up their change over the course. This can ensure that participants do not get disappointed, but - in the best casemight be positively surprised, which seems to support long-term physical activity maintenance.

Several limitations have to be mentioned. The sample of this study was comparably small and heterogeneous consisting of participants with different cancer entities and treatments. The division in four outcome realization groups yielded very small subgroups that did not allow more advanced statistics (such as changes over time) and the inclusion of control variables. However, as simple group comparisons yielded interesting outcomes which are in line with the results by previous studies among older adults (Neff and King, 1995; Wilcox et al., 2006), future studies with a bigger and more representative sample should replicate the results. A further limitation was that the study consisted of two randomly assigned interventions and in the stress management intervention physical activity was not addressed directly. However, participants of this group were kept in the analyses as previous analyses have found significant increases in physical activity also among the participants practicing stress management (Ungar et al., 2016a). Only one participant did not report any experiences with physical activity during the intervention phase and was thus excluded from analysis. In addition, the intervention group variable was not significantly related to any outcome variable or to the assignment of the four outcome realization groups. Finally, meeting physical activity guidelines was measured via selfreport and analyzed in a dichotomous way. Next to the fact that the division into (not) meeting guidelines is very relevant in practice and was the main outcome variable in the MOTIVACTION study, a very big variance in the physical activity variable with a clumping at zero did not yield robust results in a continuous analysis. The limitation of self-report can be mitigated by the fact that baseline selfreported physical activity was validated by accelerometer-based data.

In conclusion, this study revealed that cancer patients had positive expectancies toward physical activity, whereas their experiences were slightly more negative. Results corroborate the theory by Rothman (2000) that behavioral experiences with a health behavior play a key role in behavior maintenance. Our results revealed that not only initial outcome expectancies but also their realization are important for subsequent behavior and cognitions. Especially, cancer patients with comparatively low initial expectancies but better experiences were successful on three out of four outcome variables. A more fluid conceptualization of outcome expectancies (Wilcox et al., 2006) taking into account participants' behavioral experiences can better reflect dynamic changes in outcome expectancies due to a failed or successful realization. Also, future intervention studies and clinicians may focus on generating positive physical activity experiences especially if the outcome expectancies of cancer patients are low.

\section{Declaration of conflicting interests}

The author(s) declared no potential conflicts of interest with respect to the research, authorship, and/or publication of this article.

\section{Funding}

The author(s) disclosed receipt of the following financial support for the research, authorship, and/or publication of this article: The authors thank the Ernst Ludwig 
Ehrlich Scholarship Programme for supporting Nadine Ungar to conduct the MOTIVACTION study.

\section{Supplemental material}

Supplemental material for this article is available online.

\section{ORCID iD}

Nadine Ungar

4561-4228

\section{References}

Bandura A (1986) Social Foundations of Thought and Action: A Social Cognitive Theory. Upper Saddle River, NJ: Prentice-Hall.

Bandura A (1997) Self-Efficacy: The Exercise of Control. New York: Worth Publishers.

Buffart LM, Kalter J, Sweegers MG, et al. (2017) Effects and moderators of exercise on quality of life and physical function in patients with cancer: An individual patient data meta-analysis of 34 RCTs. Cancer Treatment Reviews 52: 91-104.

Cormie P, Zopf EM, Zhang X, et al. (2017) The impact of exercise on cancer mortality, recurrence, and treatment-related adverse effects. Epidemiological Reviews 39: 71-92.

Coups EJ, Park BJ, Feinstein MB, et al. (2009) Correlates of physical activity among lung cancer survivors. Psycho-Oncology 18: 395404.

Courneya KS (2009) Physical activity in cancer survivors: A field in motion. Psycho-Oncology 18: 337-342.

Crawford JJ, Holt NL, Vallance JK, et al. (2016) A new paradigm for examining the correlates of aerobic, strength, and combined exercise: An application to gynecologic cancer survivors. Supportive Care in Cancer 24: 3533-3541.

Demark-Wahnefried W, Aziz NM, Rowland JH, et al. (2005) Riding the crest of the teachable moment: Promoting long-term health after the diagnosis of cancer. Journal of Clinical Oncology 23: 5814-5830.

Endrighi R, Basen-Engquist K, Szeto E, et al. (2016) Self-reported and automatic cognitions are associated with exercise behavior in cancer survivors. Health Psychology 35: 824-828.

Fuchs R (1994) Konsequenzerwartungen als Determinante des Sport-und Bewegungsverh- altens [Outcome expectancies as a determinant of physical exercise]. Zeitschrift für Gesundheitspsychologie 2: 269-291.

Fuchs R (2013) Sportbezogene Konsequenzerfahrungen: Das Konstrukt und seine Messung [Exercise-Related Outcome Experiences: The Construct and its Operationalization]. Freiburg: Universität Freiburg.

Hoffmann TC and Del Mar C (2015) Patients' expectations of the benefits and harms of treatments, screening, and tests: A systematic review. JAMA Internal Medicine 175: 274-286.

International Agency for Research on Cancer (2017) GLOBOCAN 2012: Estimated Cancer Incidence, Mortality and Prevalence Worldwide in 2012. Lyon, France: IARC.

Klusmann V, Musculus L, Sproesser G, et al. (2016) Fulfilled emotional outcome expectancies enable successful adoption and maintenance of physical activity. Frontiers in Psychology 6: 1990.

Loehr VG, Baldwin AS, Rosenfield D, et al. (2014) Weekly variability in outcome expectations: Examining associations with related physical activity experiences during physical activity initiation. Journal of Health Psychology 19: 1309-1319.

Mishra SI, Scherer RW, Snyder C, et al. (2012) Exercise interventions on health-related quality of life for people with cancer during active treatment. The Cochrane Library. Epub ahead of print 15 August 2012. DOI:10.1002/14651858. CD008465.pub2

Neff K and King A (1995) Exercise program adherence in older adults: The importance of achieving one's expected benefits. Medicine and Exercise in Nutrition and Health 4: 355-362.

Nudelman G and Shiloh S (2018) Connectionism and behavioral clusters: Differential patterns in predicting expectations to engage in health behaviors. Annals of Behavioral Medicine 52: 890-901.

Rogers LQ, Fogleman A, Verhulst S, et al. (2015) Refining measurement of social cognitive theory factors associated with exercise adherence in head and neck cancer patients. Journal of Psychosocial Oncology 33: 467487.

Rogers LQ, Markwell S, Hopkins-Price P, et al. (2011) Reduced barriers mediated physical activity maintenance among breast cancer survivors. Journal of Sport \& Exercise Psychology 33: $235-254$. 
Rothman AJ (2000) Toward a theory-based analysis of behavioral maintenance. Health Psychology 19: 64-69.

Sallis JF, Grossman RM, Pinski RB, et al. (1987) The development of scales to measure social support for diet and exercise behaviors. Preventive Medicine 16: 825-836.

Schmitz KH, Courneya KS, Matthews C, et al. (2010) American College of Sports Medicine roundtable on exercise guidelines for cancer survivors. Medicine and Science in Sports and Exercise 42: 1409-1426.

Schwarzer R (2008) Modeling health behavior change: How to predict and modify the adoption and maintenance of health behaviors. Applied Psychology 57: 1-29.

Schwarzer R, Sniehotta FF, Lippke S, et al. (2003) On the Assessment and Analysis of Variables in the Health Action Process Approach: Conducting an Investigation. Berlin: Freie Universitaet Berlin.

Sears SR and Stanton AL (2001) Expectancyvalue constructs and expectancy violation as predictors of exercise adherence in previously sedentary women. Health Psychology 20: 326-333.

Ungar N, Sieverding M, Weidner G, et al. (2016a) A self-regulation-based intervention to increase physical activity in cancer patients. Psychology Health \& Medicine 21: 163-175.

Ungar N, Wiskemann J and Sieverding M (2016b) Physical activity enjoyment and self-efficacy as predictors of cancer patients' exercise behavior. Frontiers in Psychology 7: 898.

Vallance J and Courneya K (2012) Social cognitive approaches to understanding exercise motivation and behavior in cancer survivors. In: Roberts GC and Treasure CD (eds) Advances in Motivation in Sport and Exercise. Champaign, IL: Human Kinetics, pp. 299-326.

Wagenmakers R, van den Akker-Scheek I, Groothoff JW, et al. (2008) Reliability and validity of the short questionnaire to assess health-enhancing physical activity (SQUASH) in patients after total hip arthroplasty. BMC Musculoskeletal Disorders 9: 141.

Weeks JC, Catalano PJ, Cronin A, et al. (2012) Patients' expectations about effects of chemotherapy for advanced cancer. New England Journal of Medicine 367: 1616-1625.

Wendel-Vos GCW, Schuit AJ, Saris WHM, et al. (2003) Reproducibility and relative validity of the short questionnaire to assess healthenhancing physical activity. Journal of Clinical Epidemiology 56: 1163-1169.

Wilcox S, Castro CM and King AC (2006) Outcome expectations and physical activity participation in two samples of older women. Journal of Health Psychology 11: 65-77.

Young M, Plotnikoff R, Collins C, et al. (2014) Social cognitive theory and physical activity: A systematic review and meta-analysis. Obesity Reviews 15: 983-995. 